\title{
A Case of Bladder Flap Hematoma Presenting With Hematuria
}

\author{
Gülşah İlhan*, Fatma Ferda Verit Atmaca, Ali Galip Zebitay, Hüseyin Gültekin, Ahmet Hasan Ergin, Karolin \\ Ohanoğlu
}

\begin{abstract}
Objectives: Patients with bladder flap hematoma usually present with a mass lesion, signs of hypovolemia and/or infection. We herein introduce a patient that presented with an extraordinary sign of bladder flap hematoma.

Case Presentation: A woman with term pregnancy presented to our hospital in labor. She underwent cesarean delivery due to persistent late decelerations. Cesarean delivery was performed with the traditional cesarean section method. On the first postoperative hour, the patient developed postpartum hemorrhage due to uterine atony. She had severe anemia and tachycardia. Transvaginal ultrasonography revealed the presence of $86 \times 77 \mathrm{~mm}$ solid mass interposed between lower uterine segment and bladder. Slow-onset hematuria was noticed in the previously clear urinary discharge. The patient was treated conservatively.

Conclusion: Hematuria may be a sign of bladder flap hematoma. Though no exact and standardized protocols exist for the management, conservative approach is one of the treatment modalities and may be offered for eligible patients.

Keywords: Bladder flap, Cesarean section, Complication, Hematuria
\end{abstract}

\section{Introduction}

Bladder flap hematoma is an unusual complication of cesarean delivery. The exact incidence is unknown (1). A few paper has been written on this surgical complication $(2-5)$.

Bladder flap formation has been an important step in standard cesarean delivery. A cesarean delivery can be performed either by suturing or not suturing the visceral peritoneum. When visceral peritoneum is reapproximated, bleeding at the incision site may result in bladder flap hematoma. The traditional method closes visceral and parietal peritoneum, and therefore may lead to this complication (6).

Patients with bladder flap hematoma usually present with a mass lesion, signs of hypovolemia (tachycardia, drop in hemoglobin level, decreased urinary output) and/or infection (fever, leukocytosis) (2-5). In the present case, we introduce a patient who presented with an extraordinary sign of bladder flap hematoma.

\section{Case Presentation}

A 25-year-old nulliparous, 40 weeks and 5 days pregnant woman presented to Suleymaniye Maternity, Research and Education Hospital in labor. Transabdominal ultrasonography showed $3490 \mathrm{~g}$ fetus in cephalic presentation. She had regular contractions on non-stress test. Her cervical examination revealed a dilatation of $4 \mathrm{~cm}$ and an effacement of $80 \%$. She was hospitalized in order to perform vaginal delivery.

The woman had neither obstetric risk factors nor sys- temic diseases. Prenatal screening of the fetus was unremarkable. The patient underwent cesarean delivery due to persistent late decelerations during active labor. Cesarean delivery was performed with the technique of traditional method. The visceral peritoneum was sutured as in the traditional technique. The newborn's weight was $3220 \mathrm{~g}$ with an Apgar score of 7 at 1 minute and 9 at 5 minutes. On the first postoperative hour, the patient developed postpartum hemorrhage due to uterine atony. She had severe anemia and tachycardia. Transvaginal ultrasonography revealed the presence of $86 \times 77 \mathrm{~mm}$ solid mass interposed between lower uterine segment and bladder (Figure 1). Preoperative hemoglobin level was $11 \mathrm{mg} / \mathrm{dL}$ and postoperative drop in hemoglobin was $5 \mathrm{mg} / \mathrm{dL}$. Although bleeding time (2 minutes), platelet count $(165.000 / \mathrm{mcL})$, and fibrinogen level $(310 \mathrm{mg} / \mathrm{dL})$ were normal, slow-onset hematuria was noticed in the previously clear urinary discharge. She was transfused with 4 units of erythrocyte suspension and 4 units of fresh frozen plasma. The follow ups of the patient were performed using serial transabdominal ultrasounds. The bladder flap hematoma was stable in dimension and the patient was clinically asymptomatic. Intravenous antibiotic therapy with ampicillin/sulbactam (4 g/day) was ordered. Urinary catheter was not removed until gross hematuria was resolved. She had no fever or leukocytosis during the follow-up period. Transvaginal ultrasonography on seventh post-operative day showed an obvious reduction in hematoma size $(32 \times 33 \mathrm{~mm})$ (Figure 2). The patient was discharged with oral ampicillin/ sulbactam (1.5 g/day) on the seventh post-operative day 
and outpatient clinic control was advised.

Examination of patient on 14th post-operative day showed complete resolution of the hematoma. The patient has been following up and have had no complaints on postoperative ninth month.

\section{Discussion}

Cesarean delivery is the most common major surgical operation in women worldwide (7). It is definitely important to use the most effective and the safest operation technique. Despite the fact that cesarean delivery is a routine surgical procedure, an unanimity on the most appropriate operative technique to use have not yet been concluded. Standardized, evidence-based techniques are imperative to minimize complications of the operation.

The traditional method is one of the most commonly used cesarean delivery techniques (8). In this method, visceral and parietal peritoneum is closed respectively and a potential space for blood collection is formed. Bladder flap hematoma is formed as a result of bleeding at the incision site and located between urinary bladder and lower uterine segment. The source of bleeding is the injury of uterine vessels (5). In a cesarean section performed with Misgav Ladach or Stark method by not suturing the visceral peritoneum, the vesico-uterine space communicates with the large peritoneal cavity and this may prevent the formation of a bladder flap hematoma (9).

The exact incidence of this complication is unknown. Burger et al (1) showed the sonographic evaluation of 48 patients operated with low transverse cesarean delivery. Fourteen of them had fluid collection between the bladder and uterus but none of the patients had any symptoms. The diagnosis of bladder flap hematoma is based on a mass between bladder and uterus in the postoperative patient presenting with drop in hematocrit level or fever. Ultrasonographic evaluation shows a well-circumferenced complex collection located between bladder and uterus causing posterior shadowing (2). In our case, cesarean delivery was performed by traditional method. Initial symptoms of the patient was vaginal bleeding, tachycardia and drop in hemoglobin level. Transvaginal ultrasonography revealed the presence of well-circumferenced, solid mass interposed between lower uterine segment and bladder. It

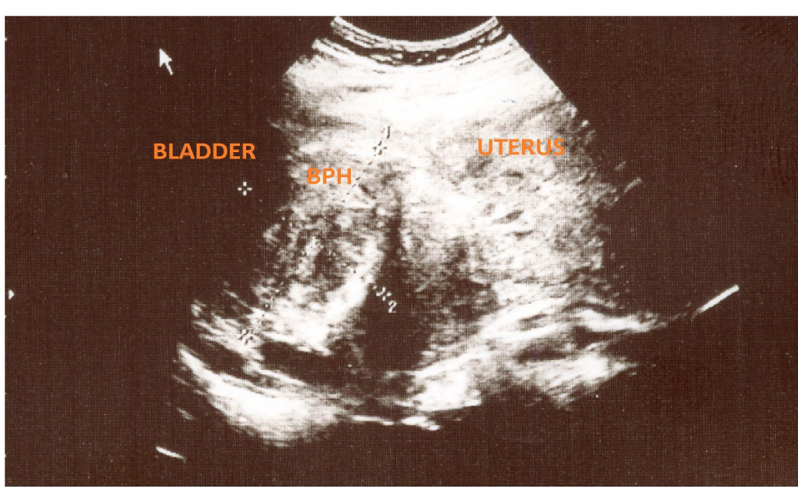

Figure 1. Transvaginal Ultrasonography of Bladder Flap Hematoma at Postoperative First Hour. BPH: bladder flap hematoma. was thought that decreased uterine tonus has led to bleeding of the Kerr incision and resulted in bladder flap hematoma. During the following hours, fusion of the blood through the bladder was trigger reason of hematuria.

The scientific literature have no clear and defined protocol for clinical management, or for its surgical treatment. Surgical management of symptomatic postcesarean section bladder flap hematoma, include laparoscopic-laparotomic drainage, percutaneous ultrasound or computed tomography-guided aspiration (10), surgical transvaginal evacuation, and additive obliteration therapy (injection of a sealant such as an antibiotic doxycycline into the hematoma) $(9,11)$.

Transabdominal or transvaginal ultrasound-guided drainage and additive obliteration therapy in these circumstances can be performed safely, avoids further surgery and might cause only little discomfort to the patient. Baker et al (5) reported seven patients with bladder flap hematoma in 1985. All sonographies demonstrated characteristic localisation. Postoperative hemoglobin drop ranged between $1.3-6 \mathrm{mg} / \mathrm{dL}$ in this case series. One patient underwent hysterectomy and another had exploratory laparotomy. Five of the patients were treated conservatively in this case series.

Winsett et al (2) reported 10 cases of bladder flap hematoma in 1986. Bladder flap hematoma resulted from primary low transverse cesarean section in six patients. In four patients, a low transverse cesarean section with classic extension, a transabdominal hysterectomy with bilateral salpingo-oophorectomy, a transvaginal hysterectomy, a right salpingo-oophorectomy with left salpingectomy were performed, respectively. Ultrasonography demonstrated a mass lesion in the characteristic localisation. Hematoma was treated surgically in five patients and the other five patients were treated with antibiotic alone.

Tinelli et al (4) reported two cases of bladder flap hematoma in 2007. These cases were submitted to laparoscopic treatment. Tinelli et al (3) in 2009 reported 10 symptomatic women undergoing laparoscopy. The essential steps of the operation were expressed as incision of the tumescence, bladder wall dissection from the vesico-uterine space, drainage of the collection, washing of the hemato-

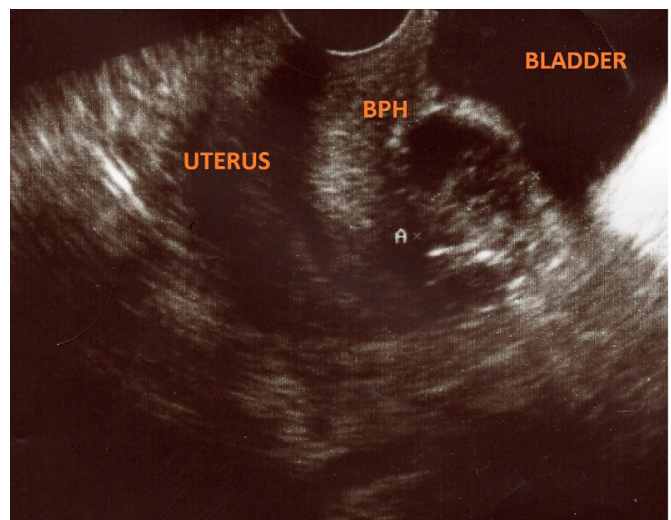

Figure 2. Transvaginal Ultrasonography of Bladder Flap Hematoma on Seventh Postoperative Day. BPH: bladder flap hematoma. 
ma site and peritoneal suturing.

As mentioned, no standardized treatment for bladder flap hematoma exists in the literature. It might be deduced that antibiotic therapy is the first treatment step and surgery may be considered in cases with infection or bleeding. In our study; the patient was treated conservatively. We started intravenous antibiotic therapy with ampicillin/sulbactam (4 g/day). She had no fever or leukocytosis during the follow-up period. Transvaginal ultrasonography on seventh post-operative day showed an obvious reduction in hematoma size $(32 \times 33 \mathrm{~mm})$. This obvious reduction of hematoma size might be explained by fusion of the blood through the bladder and resulted in hematuria. The slow-onset hematuria of the patient might be explained in this way.

In conclusion, bladder flap hematomas are unusual complications of a standard cesarean delivery. Their detection using sonography is an easy and accurate way. However, hematuria was not mentioned in any of the above mentioned cases, it may be a sign of bladder flap hematoma. Conservative approach is one of the treatment modalities and may be offered for patients in stable clinical status.

\section{Ethical Issues}

The authors have obtained permission before using patient data and images.

\section{Conflict of Interests}

Authors declare that they have no competing interests.

\section{Financial Support}

None.

\section{Acknowledgments}

None to be declared.

\section{References}

1. Burger NF, Darazs B, Boes EG. An echographic evaluation during the early puerperium of the uterine wound after caesarean section. J Clin Ultrasound 1982;10(6):271-4. doi: 10.1002/jcu.1870100605.

2. Winsett MZ, Fagan CJ, Bedi DG. Sonographic demonstration of bladder-flap hematoma. J Ultrasound Med. 1986;5(9):483-7.

3. Tinelli A, Malvasi A, Vittori G. Laparoscopic treatment of post-cesarean section bladder flap hematoma: A feasible and safe approach. Minim Invasive Ther Allied Technol. 2009;18(6):356-60. doi: 10.3109/13645700903201357.

4. Tinelli A, Malvasi A, Tinelli R, Cavallotti C, Tinelli FG. Conservative laparoscopic treatment of postcaesarean section bladder flap haematoma: two case reports. Gynecol Surg. 2007;4(1):53-6. doi: 10.1007/ s10397-006-0212-2

5. Baker ME, Bowie JD, Killam AP. Sonography of post-cesarean-section bladder-flap hematoma. AJR Am J Roentgenol. 1985;144(4):757-9. doi: 10.2214/ ajr.144.4.757

6. Bamigboye AA, Hofmeyr GJ. Closure versus nonclosure of the peritoneum at caesarean section. Cochrane Database Syst Rev. 2014;(8):CD000163. doi: 10.1002/14651858.CD000163.

7. Hamilton BE, Martin JA, Ventura SJ. Births: Preliminary data for 2008. Natl Vital Stat Rep. 2010;59(3):1-19. doi: 10.13140/RG.2.1.2768.4000

8. Vitale SG, Marilli I, Cignini P, et al. Comparison between modified Misgav-Ladach and PfannenstielKerr techniques for cesarean section: review of literature. J Prenatal Med. 2014;8(3-4):36-41.

9. Malvasi A, Tinelli A, Tinelli R, Rahimi S, Resta L, Tinelli FG. The post cesarean section symptomatic bladder flap haematoma: a modern appraisal. J Matern Fetal Neonatal Med. 2007;20(10):709-14. doi: 10.1080/01674820701450573.

10. Achonolu F, Minkoff H, Delke I. Percutaneous drainage of fluid collections in the bladder flap hematoma of febrile postcaesarean section patients. A report of seven cases. J Reprod Med. 1987;32(2):1403.

11. Winter TC, Lee FT Jr, Hinshaw JL. Ultrasoundguided biopsies in the abdomen and pelvis. Ultrasound Q. 2008;24(1):45-68. doi: 10.1097/ RUQ.0b013e318168c869.

Copyright $\odot 2016$ The Author(s); This is an open-access article distributed under the terms of the Creative Commons Attribution License (http://creativecommons.org/licenses/by/4.0), which permits unrestricted use, distribution, and reproduction in any medium, provided the original work is properly cited. 